\title{
Werkzeuge gegen kognitive Verzerrungen im klinischen Alltag
}

\section{Marko Kovic}

Dr. phil., Publizistik- und Kommunikationswissenschaft, CEO der ars cognitionis $\mathrm{GmbH}$, Zürich

\section{Denkfehler und ihre Folgen}

Der klinische Alltag ist anspruchsvoll. Unter oftmals schwierigen Bedingungen (zu wenig Personal, zu wenig Zeit, zu wenig Schlaf) müssen Ärztinnen und Ärzte wie auch das gesamte medizinische Fachpersonal viele und weitreichende Entscheidungen treffen. Wo Men-

\section{Wo Menschen unter widrigen Bedingungen Entscheidungen treffen müssen, passieren auch Fehler.}

schen unter widrigen Bedingungen Entscheidungen treffen müssen, passieren auch Fehler. Gemäss Schätzungen der OECD etwa sind rund 10\% der gesamten Spitalkosten auf vermeidbare diagnostische oder therapeutische Fehler zurückzuführen [1]. Wenn in der klinischen Praxis Fehler passieren, bedeutet das nicht, dass die betroffenen Ärztinnen und Ärzte über zu wenig Fachkompetenz verfügen. Die Quelle des Grossteils von Fehlern im klinischen Alltag ist nämlich nicht mangelnde Fachkompetenz, sondern die allgemeine menschliche Anfälligkeit für kognitive Verzerrungen.

Kognitive Verzerrungen sind Denkfehler, welche unbemerkt auftreten, weil wir einen grossen Teil unserer

\section{Résumé}

Les erreurs de raisonnement routinières, appelées biais cognitifs, influencent la qualité de la prise de décision dans tous les domaines sociaux, y compris la médecine. Dans le quotidien clinique, elles sont à l'origine de nombreuses erreurs diagnostiques et thérapeutiques. II existe deux stratégies pour réduire l'impact négatif des biais cognitifs: le debiasing et le nudging. Le debiasing a pour but de réduire de manière ciblée la tendance individuelle aux biais cognitifs. Le nudging vise à adapter le contexte d'action aux biais cognitifs en sorte que le comportement souhaité devienne plus probable. En tant que mesures de gestion de la qualité, le debiasing et le nudging peuvent avoir des effets positifs durables. Et cela d'autant plus que ces méthodes permettent d'améliorer la prise de décision du personnel médical, mais aussi le comportement des patients ou des visiteurs de l'hôpital, par exemple.
Denkarbeit in einem automatisierten Modus abspulen [2, 3]: Wir treffen Entscheidungen meistens nicht nach ausführlichem und langsamen Überlegen, sondern eher möglichst rasch und effizient. Wenn wir uns in diesem eher raschen Denkmodus befinden, bedienen wir uns ganz automatisch kognitiver Abkürzungen, sogenannter Heuristiken. Kognitive Heuristiken sind in vielen Situationen gut genug, um rationale, also zielführende Entscheidungen treffen. In komplexeren Entscheidungssituationen können uns Heuristiken aber auch zu Schlussfolgerungen und Entscheidungen führen, die weit davon entfernt sind, rational zu sein. In solchen Situationen haben Heuristiken also einen verzerrenden Effekt auf unsere Entscheidungsfindung, weswegen sie pejorativ als kognitive Verzerrungen bezeichnet werden. Kognitive Verzerrungen treten auch

Wir treffen Entscheidungen meistens nicht nach ausführlichem und langsamem Überlegen, sondern eher möglichst rasch und effizient.

im klinischen Alltag auf [4-6], weil der menschliche Denkapparat ohne Heuristiken nicht auskommt überall und immer, wenn Menschen Entscheidungen treffen, treten kognitiven Verzerrungen auf.

Kognitive Verzerrungen machen sich im klinischen Alltag auf ganz unterschiedliche Art und Weise bemerkbar $[7,8]$. Sie sind dabei kein Randphänomen, sondern ein sehr zentrales und folgenreiches Problem. So sind einige prominente Verzerrungen wie etwa Anchoring (die erste Information, welche wir erhalten, beeinflusst, wie wir weitere Informationen bewerten), Confirmation Bias (wir versuchen das, woran wir bereits glauben, zu bestätigen), Overconfidence (wir haben ein zu hohes Vertrauen in die Qualität unserer Entscheidungen) oder Availability Bias (wir orientieren uns bei Schlussfolgerung zu sehr an einprägsamen Bildern und Beispielen, die wir gerade im Kopf haben) bei der Mehrheit aller Ärztinnen und Ärzte zu beobachten [9]. Kognitive Verzerrungen als alltägliches Problem nimmt noch grössere Dimensionen an, wenn wir bedenken, dass im klinischen Alltag nicht nur Ärztinnen und Ärzte Entscheidungen treffen: Das weitere medi- 
zinische Fachpersonal, nicht-medizinisches Personal (z.B. das Spitalmanagement), Besucherinnen und Besucher und nicht zuletzt auch Patientinnen und Patienten sind von kognitiven Verzerrungen betroffen.

Kognitive Verzerrungen haben also einen beträchtlichen negativen Impact im klinischen Alltag. Dieser negative Impact kann aber reduziert werden: Es gibt Strategien, um kognitiven Verzerrungen entgegenzuwirken.

\section{Gegen Denkfehler vorgehen: Debiasing und Nudging}

Kognitive Verzerrungen sind der normale Modus Operandi unseres menschlichen Denkapparates. Die Forschung zu kognitiven Verzerrungen zeigt aber auch, dass es möglich ist, etwas gegen sie zu unternehmen. Es gibt es zwei erfolgversprechende evidenzbasierte Strategien, welche als Werkzeuge gegen kognitive Verzerrungen eingesetzt werden können: Debiasing und Nudging.

\section{Debiasing}

Debiasing bedeutet ganz allgemein, dass versucht wird, die individuelle Anfälligkeit für kognitive Verzerrungen zu reduzieren. Im medizinischen Kontext wird Debiasing bisweilen unter dem Stichwort «Cognitive Forcing" diskutiert [10]. Cognitive Forcing, zu Deutsch so viel wie kognitives Erzwingen, meint die

\section{Es gibt Strategien, um kognitiven Verzerrungen entgegenzuwirken.}

Anstrengung, in Entscheidungssituationen aktiv über das eigene Denken zu denken. Debiasing im Sinne von Cognitive Forcing ist also eine sogenannte metakognitive Aktivität, welche zum Ziel hat, aus dem automatisierten Denkmodus in einen bedachteren und begründeten Denkmodus zu wechseln. Cognitive Forcing bedeutet in erster Linie, dass Know-how rund um kognitive Verzerrungen hilft, weniger anfällig auf kognitive Verzerrungen zu sein [11]: Je besser z.B. Ärztinnen und Ärzte über kognitive Verzerrungen Bescheid wissen, desto weniger anfällig werden sie dafür. Debiasing-Strategien im klinischen Kontext können aber auch in Richtung kognitiver Hilfsmittel gehen [12]: In konkreten Entscheidungssituationen helfen kognitive Hilfsmittel, das schnelle, routinisierte Denken zu einem überlegteren Denken zu wandeln. Wirksame kognitive Hilfsmittel sind beispielsweise die «erwäge das Gegenteil»-Strategie (aktiv überlegen, was für das Gegenteil der Schlussfolgerung z.B. bei einer Diagnose spricht) oder das gezielte Verlangsamen von Entscheidungsprozessen (z.B. im Voraus geplante
Pausen, um den Denk- und Entscheidungsprozess zu verlangsamen). Eines der effektivsten kognitiven Hilfsmittel sind Checklisten. Checklisten können als unmittelbares Kontrollmittel in komplexen Prozessen eingesetzt werden, um Fehler zu vermeiden. Checklisten können aber auch als übergeordnetes DebiasingWerkzeug eingesetzt werden, um einzelne kognitive Hilfsmittel wie etwa die «erwäge das Gegenteil»-Strategie oder Verlangsamungsstrategien bewusst einzusetzen [13].

\section{Nudging}

Nudging ist das englische Wort für Stupser. Während Debiasing zum Ziel hat, die Anfälligkeit für kognitive Verzerrungen zu senken, bedeutet Nudging, dass kognitive Verzerrungen bewusst ausgenutzt werden. Wenn wir einschätzen können, in welchen Entscheidungssituationen Menschen im klinischen Kontext für kognitive Verzerrungen anfällig sind, dann können wir den Entscheidungskontext so anpassen, dass sich Menschen eher so verhalten, wie wir es wollen. Nudging senkt also kognitive Verzerrungen nicht, sondern nutzt sie aus, damit das Verhalten insgesamt besser wird. Ein berühmtes Beispiel für Nudging ist die aufgemalte Fliege im Pissoir, welche dazu führt, dass Herren beim Urinieren genauer zielen und dadurch den Boden weniger verunreinigen. Nudging wird in zahlreichen Kontexten verwendet und zeitigt mit wenig Aufwand grossen Impact [14, 15]. Im klinischen Kontext ist Nudging bisher noch weniger erprobt als Debiasing. Das Potenzial von Nudging im klinischen Kontext liegt nicht zuletzt darin, dass damit auch das Verhalten von Patientinnen und Patienten sowie von Besucherinnen und Besuchern optimiert werden kann (Debiasing zielt in erster Linie auf das medizinische Fachpersonal ab). Ein möglicher Einsatzbereich für Nudging ist Spitalhygiene. Nudging-Massnahmen könnten helfen, hygienisch wünschenswertes Verhalten wie die Hände-Desinfektion bei Spitalbesuchern mit leichten Anpassungen des Handlungskontextes zu verbessern. Zum Beispiel können auf dem Boden subtile Markierungen angebracht werden, welche die

Debiasing bedeutet ganz allgemein, dass versucht wird, die individuelle Anfälligkeit für kognitive Verzerrungen zu reduzieren.

Besucher sanft in Richtung Desinfektions-Stationen lenken. Auf der Patientenseite ist ein möglicher Einsatzbereich das Problem der Patientenadhärenz [16]. Therapeutische Anweisungen diszipliniert und vollständig einzuhalten und umzusetzen, ist für Patienten oftmals nicht einfach. Nudging-Strategien wie z.B. Ga- 


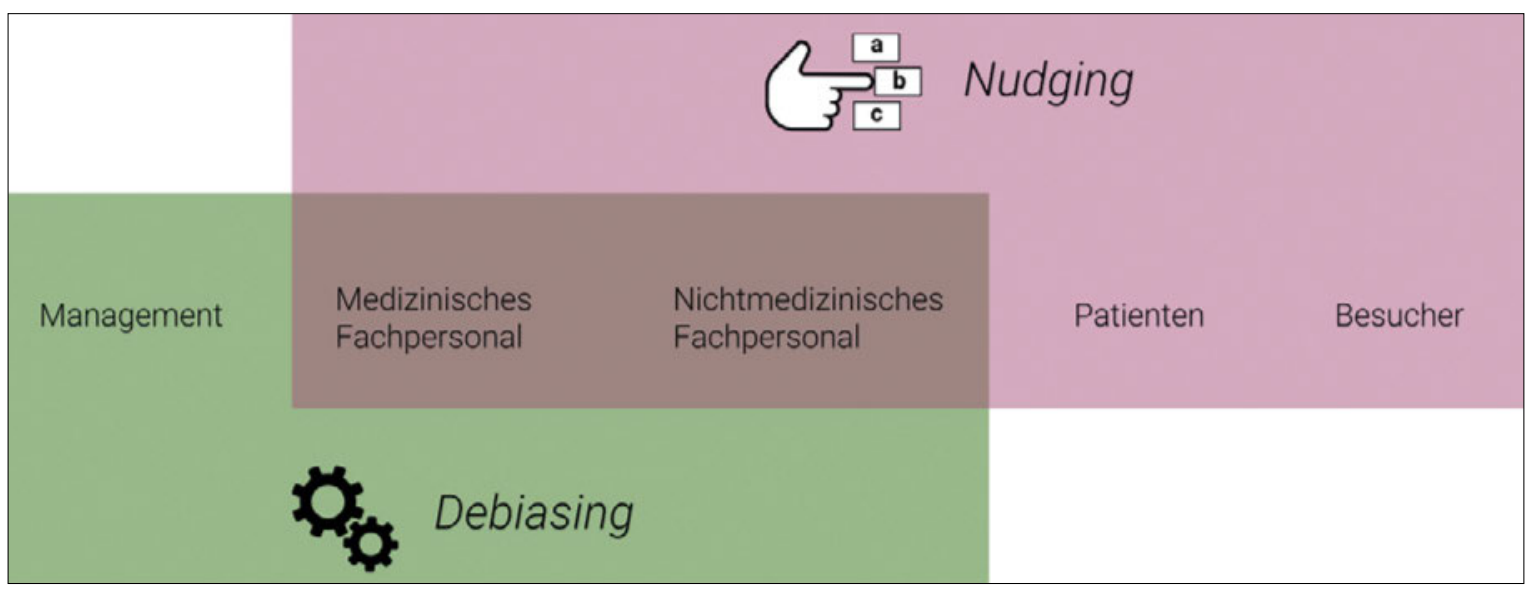

Abbildung 1: Anwendungsbereiche von Debiasing und Nudging im klinischen Kontext.

mification mit einer Smartphone-App können potenziell helfen, die Adhärenz zu verbessern, indem Patienten spielerisch an die notwendigen Schritte erinnert werden und für ihre Durchführung belohnt werden.

\section{Eine Frage der Evidenz}

Fehler und Fehlverhalten im klinischen Kontext können durch Debiasing und Nudging nicht komplett

\section{Im medizinischen Kontext müssen sowohl Debiasing wie auch Nudging explizit evidenz- basiert sein.}

eliminiert werden. Debiasing und Nudging als Werkzeuge können aber mit verhältnismässig geringem Aufwand potenziell grossen und nachhaltigen positiven Impact haben. Nicht zuletzt, weil die Einsatzbereiche von Debiasing und Nudging nicht auf das medizinische Fachpersonal beschränkt sind, sondern auf weitere Beteiligte im klinischen Alltag ausgeweitet werden können, wie in Abbildung 1 zusammengefasst ist.

Debiasing und Nudging bergen grosses Potenzial für den klinischen Kontext, aber gleichzeitig ist auch Vorsicht geboten. Die Thematik der Biases und des Nudging (sowie in geringerem Masse auch des Debiasing), ein ursprünglich angelsächsischer Trend, wird von immer mehr Akteuren in Kontinentaleuropa aufgegriffen, was grundsätzlich begrüssenswert ist. Wissenschaftliche Evidenz und ein wissenschaftlich fundiertes Vorgehen bleiben dabei allerdings allzu oft auf der Strecke, nicht zuletzt in der Beratungsbranche. Im medizinischen Kontext müssen sowohl Debiasing wie auch Nudging explizit evidenzbasiert sein und bei eher experimentellen Massnahnahmen muss der Impact der Massnahmen auch wissenschaftlich evaluiert werden. Dann, und nur dann, können Debiasing und
Nudging einen wirklich nachhaltigen positiven Impact haben und ein Teil des klinischen Qualitätsmanagements werden.

Bildnachweis

Grafik Marko Kovic

\section{Literatur}

1 Tackling Wasteful Spending on Health. OECD Publishing, Paris, January 2017. DOI: 10.1787/9789264266414-en.

2 Amos Tversky and Daniel Kahneman. Judgment under Uncertainty: Heuristics and Biases. In Utility, Probability, and Human Decision Making, Theory and Decision Library, pages 141-62. Springer, Dordrecht, 1975. DOI: 10.1007/978-94-010-1834-0 8.

3 Thierry Pelaccia, Jacques Tardif, Emmanuel Triby, and Bernard Charlin. An analysis of clinical reasoning through a recent and comprehensive approach: the dual-process theory. Medical Education Online. 2011;16(1):5890.

4 Geoffrey R. Norman and Kevin W. Eva. Diagnostic error and clinical reasoning. Medical Education. 2010;44(1):94-100.

5 Shyam S. Kothari. Clinical errors. Ann Pediatr Cardiol. 2012;5(1):1-2.

6 Ian A. Scott. Errors in clinical reasoning: causes and remedial strategies. BMJ. 2009;338:b1860.

7 Scott O. Lilienfeld and Steven Jay Lynn. Errors/Biases in Clinical Decision Making. In The Encyclopedia of Clinical Psychology. John Wiley \& Sons, Inc., 2014. DOI: 10.1002/9781118625392.wbecp567.

8 Arthur S. Elstein. Heuristics and biases: selected errors in clinical reasoning. Academic Medicine: Journal of the Association of American Medical Colleges. 1999;74(7):791-4.

9 Gustavo Saposnik, Donald Redelmeier, Christian C. Ruff, and Philippe N. Tobler. Cognitive biases associated with medical decisions: a systematic review. BMC Medical Informatics and Decision Making, 16, November 2016.

10 Pat Croskerry. Cognitive forcing strategies in clinical decisionmaking. Ann Emerg Med. 2003;41(1):110-20.

11 Pat Croskerry. From Mindless to Mindful Practice - Cognitive Bias and Clinical Decision Making. N Engl J Med. 2013;368(26):2445-8.

12 Pat Croskerry, Geeta Singhal, and Sílvia Mamede. Cognitive debiasing 2: impediments to and strategies for change. BMJ Quality \& Safety, pages bmjqs-2012-001713, August 2013.

13 John W. Ely, Mark L. Graber, and Pat Croskerry. Checklists to Reduce Diagnostic Errors. Acad Med. 2011;86(3):307.

14 Richard H. Thaler and Cass R. Sunstein. Nudge: Improving Decisions About Health, Wealth, and Happiness. Penguin Books, New York, revised \& expanded edition edition, February 2009.

15 On Amir and Orly Lobel. Stumble, Predict, Nudge: How Behavioral Economics Informs Law and Policy. Columbia Law Review. 2008;108(8):2098-137.

16 Leslie R. Martin, Summer L. Williams, Kelly B. Haskard, M. Robin DiMatteo. The challenge of patient adherence. Ther Clin Risk Manag. 2005;1(3):189-99. 